Linköping Studies in Science and Technology

Dissertations, No. 1593

\title{
Iterative Methods for Solving the Cauchy Problem for the Helmholtz Equation
}

Lydie Mpinganzima

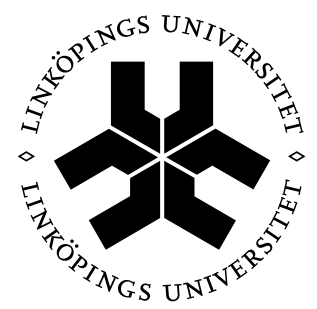

\section{Linköping University}

\section{INSTITUTE OF TECHNOLOGY}

Department of Mathematics

Linköping University, SE-581 83 Linköping, Sweden

Linköping 2014 
Iterative Methods for Solving the Cauchy Problem for the Helmholtz Equation

Copyright (C) 2014 Lydie Mpinganzima

Matematiska institutionen

Linköpings universitet

SE-581 83 Linköping, Sweden

lydie.mpinganzima@liu.se

Linköping Studies in Science and Technology

Dissertations, No. 1593

LIU-TEK-LIC-2012:15

ISBN 978-91-7519-350-2

ISSN 0345-7524

Printed by LiU-Tryck, Linköping 2014 


\section{Abstract}

The inverse problem of reconstructing the acoustic, or electromagnetic, field from inexact measurements on a part of the boundary of a domain is important in applications, for instance for detecting the source of acoustic noise. The governing equation for the applications we consider is the Helmholtz equation. More precisely, in this thesis we study the case where Cauchy data is available on a part of the boundary and we seek to recover the solution in the whole domain. The problem is ill-posed in the sense that small errors in the Cauchy data may lead to large errors in the recovered solution. Thus special regularization methods that restore the stability with respect to measurements errors are used.

In the thesis, we focus on iterative methods for solving the Cauchy problem. The methods are based on solving a sequence of well-posed boundary value problems. The specific choices for the boundary conditions used are selected in such a way that the sequence of solutions converges to the solution for the original Cauchy problem. For the iterative methods to converge, it is important that a certain bilinear form, associated with the boundary value problem, is positive definite. This is sometimes not the case for problems with a high wave number.

The main focus of our research is to study certain modifications to the problem that restore positive definiteness to the associated bilinear form. First we add an artificial interior boundary inside the domain together with a jump condition that includes a parameter $\mu$. We have shown by selecting an appropriate interior boundary and sufficiently large value for $\mu$, we get a convergent iterative regularization method. We have proved the convergence of this method. This method converges slowly. We have therefore developed two conjugate gradient type methods and achieved much faster convergence. Finally, we have attempted to reduce the size of the computational domain by solving well-posed problems only in a strip between the outer and inner boundaries. We demonstrate that by alternating between Robin and Dirichlet conditions on the interior boundary, we can get a convergent iterative regularization method. Numerical experiments are used to illustrate the performance of the methods suggested.

\section{Acknowledgements}

I take this opportunity to express my gratitude to my supervisors Vladimir Kozlov, Bengt Ove Turesson and Fredrik Berntsson for the guidance, encouragement, patience and good collaboration. Thanks also to Björn Textorius for all the discussions on various subjects in mathematics. Thanks to Martin Singull and Johan Thim for Latex templates, and the Department of Mathematics at Linköping Universtiy for providing a good working environment.

My studies have been supported by the Swedish International Development Cooperation Agency (Sida) and the University of Rwanda. I am grateful for that.

Linköping, Mars 31, 2014

Lydie Mpinganzima 


\section{Populärvetenskaplig sammanfattning}

Det inversa problemet att rekonstruera akustiska eller elektromagnetiska fält i ett område från inexakta mätningar på en del av områdets rand är viktigt för att t.ex. detektera källan för akustiskt brus. Sådana problem beskrivs av Helmholtz ekvation.

I avhandlingen studerar vi sådana problem där Cauchydata är givna endast på en del av områdets rand och man vill finna lösningen i hela området. Vi vill alltså lösa Cauchyproblem för Helmholtz ekvation. Sådana problem är illa ställda, vilket innebär att små fel i Cauchydata kan medföra stora fel i lösningen. Särskilda regulariseringsmetoder måste därför användas för att ge stabilitet med avseende på mätfel.

Avhandlingens syfte är att utveckla iterativa lösningsmetoder för dessa Cauchyproblem. Metoderna går ut på att man löser av en följd av välställda randvärdesproblem för den ursprungliga ekvationen, där randvillkoren väljs så att följden av lösningar konvergerar mot lösningen till det ursprungliga Cauchyproblemet. För att de iterativa metoderna skall konvergera är det viktigt att en viss bilinjär form, som ges av randvärdesproblemet, är positivt definit. Detta villkor är inte alltid uppfyllt för problem med högt vågtal.

I avhandlingens fokus står därför studiet av sådana modifieringar av problemet att den associerade bilinjära formen är positivt definit. Vi lägger först till en artificiell inre rand i området tillsammans med ett språngvillkor, som innehåller en parameter $\mu$. Vi visar att man genom att välja den inre randen och parametern lämpligt får en konvergent iterativ regulariseringsmetod. Konvergensen är långsam, och vi utvecklar därför i stället två metoder av konjugerad gradienttyp, vilka ger mycket snabbare konvergens.

Slutligen minskar vi beräkningsområdets storlek genom att lösa de välställda problemen endast i strimman mellan den yttre och den inre randen och visar att man genom att växla mellan Robin och Dirichletvillkor på den inre randen får en konvergent iterativ regulariseringsmetod. Numeriska experiment illustrerar de föreslagna metoderna. 


\section{List of Papers}

The thesis contains three articles and a technical report:

0. F. Berntsson, V.A. Kozlov, L. Mpinganzima, and B.O. Turesson, Numerical solution for the Cauchy problem for the Helmholtz equation, Technical report, LiTH-MAT-R-2014/04-SE, Department of Mathematics, Linköping University.

1. F. Berntsson, V.A. Kozlov, L. Mpinganzima, and B.O. Turesson, An alternating iterative procedure for the Cauchy problem for the Helmholtz equation, Inverse Problems in Science and Engineering 22(2014), No. 1, 45-62.

2. F. Berntsson, V.A. Kozlov, L. Mpinganzima, and B.O. Turesson, An accelerated alternating iterative procedure for the Cauchy problem for the Helmholtz equation, submitted.

3. F. Berntsson, V.A. Kozlov, L. Mpinganzima, and B.O. Turesson, Robin-Dirichlet algorithms for the Cauchy problem for the Helmholtz equation, manuscript. 


\section{Contents}

Introduction 1

1 The alternating iterative method 3

2 Summary of papers 4

Paper 0: Numerical solution for the Cauchy problem for the Helmholtz equation 15

$\begin{array}{llr}1 & \text { Introduction } & 15\end{array}$

2 An ill-posed operator equation $\quad 17$

2.1 The operator equation . . . . . . . . . . . . . . . . . 17

2.2 A concrete test problem . . . . . . . . . . . . . 18

3 Numerical implementation $\quad \mathbf{2 0}$

3.1 The Helmholtz equation in a rectangle . . . . . . . . . . . . . . 20

3.2 The matrix approximation . . . . . . . . . . . . . . . . 21

4 Numerical study of the Helmholtz equation 22

5 An overview of regularization methods $\quad 22$

5.1 Direct regularization methods . . . . . . . . . . . . . . . . 23

5.2 Iterative regularization methods . . . . . . . . . . . . . . 26

6 Concluding Remarks 28

Paper 1: An alternating iterative procedure for the Cauchy problem for the Helmholtz equation 33

1 Introduction $\quad 33$

1.1 The Helmholtz equation . . . . . . . . . . . . . . . . . . . 33

1.2 The alternating algorithm . . . . . . . . . . . . . . 35

1.3 Non-convergence of the standard algorithm . . . . . . . . . . . 36

1.4 A modified alternating algorithm . . . . . . . . . . . . . . 37

2 Bilinear form and properties of traces $\quad 38$

2.1 Function spaces . . . . . . . . . . . . . . . . . . 38

2.2 A bilinear form $a_{\mu}$ and a sufficient condition for $a_{\mu}$ to be positive

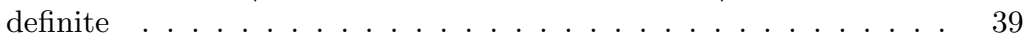

2.3 Traces and their properties . . . . . . . . . . . . . 41

3 The main theorem $\quad 43$

4 Numerical results $\quad 46$ 
Paper 2: An accelerated alternating procedure for the Cauchy problem for the Helmholtz equation

1 Introduction $\quad \mathbf{5 7}$

1.1 The Helmholtz equation . . . . . . . . . . . . . . . . . . 57

1.2 The modified alternating algorithm . . . . . . . . . . . 58

2 Preliminaries $\quad 61$

2.1 Function spaces . . . . . . . . . . . . . . . . . . . . . . 61

2.2 Weak solutions . . . . . . . . . . . . . . . . . 62

2.3 Inner products . . . . . . . . . . . . . . . . . . 63

3 Two operator equations $\quad 63$

3.1 The first operator equation . . . . . . . . . . . . . 63

3.2 The second operator equation . . . . . . . . . . . . . . 65

3.3 Stopping rule for the modified algorithm . . . . . . . . . . . . 67

$\begin{array}{lll}4 & \text { Conjugate gradient methods } & \mathbf{6 7}\end{array}$

4.1 The conjugate gradient method $(\mathrm{CGNE}) \ldots \ldots$. . . . . . . . . 67

4.2 Stopping rule for CGNE . . . . . . . . . . . . . . . . 68

4.3 The minimal error method $(\mathrm{CGME}) \ldots \ldots \ldots$

4.4 Stopping rule . . . . . . . . . . . . . . . . . . . . 70

5 Numerical results $\quad \mathbf{7 1}$

5.1 Numerical discretization . . . . . . . . . . . . . . . . . 71

5.2 Numerical tests . . . . . . . . . . . . . . . . . . 73

5.3 Discussions . . . . . . . . . . . . . . . . . 77

$\begin{array}{llr}6 & \text { Conclusions } & 79\end{array}$

Paper 3: Robin-Dirichlet algorithms for the Cauchy problem for the $\begin{array}{ll}\text { Helmholtz equation } & 87\end{array}$

$\begin{array}{llr}1 & \text { Introduction } & 87\end{array}$

$\begin{array}{llr}2 & \text { Preliminaries } & 89\end{array}$

3 Description of the algorithm $\quad 89$

3.1 The first algorithm . . . . . . . . . . . . . . . . . . . . . 89

3.2 The second algorithm $\ldots \ldots \ldots$. . . . . . . . . . . . . . . . 91

4 Sufficient condition for $a_{\mu}$ to be positive definite $\quad 92$

4.1 Example . . . . . . . . . . . . . . . . . . . 92

4.2 Validity of relation (3.4) in a two dimensional case . . . . . . . . 93

5 Numerical experiments $\quad \mathbf{9 4}$

5.1 Finite difference approximation . . . . . . . . . . . . . . . 95

5.2 Numerical tests and discussions . . . . . . . . . . . . . . . . 97

6 Conclusions 100 


\section{Introduction}

The Helmholtz equation arises in a wide range of applications related to acoustic and electromagnetic waves. Depending on the type of the boundary conditions, it is involved in the determination of acoustic cavities [14], the detection of the source of acoustical noise [17], the description of underwater waves [18], the determination of the radiation field surrounding a source of radiation [15], the localization of a tumor in a human body [16], the identification and location of vibratory sources [23], the detection of surface vibrations from interior acoustical pressure [20], etc.

In this paper, we consider the inverse problem of reconstructing the acoustic or electromagnetic field from inexact data given only on an open part of the boundary of a given domain. The governing equation for such problem is the Helmholtz equation. This problem is known as the Cauchy problem for the Helmholtz equation and it is ill-posed. According to Hadamard's definition of well-posedness, a problem is well-posed if it satisfies the following three requirements; (see [19]):

1. Existence: There exists a solution of the problem.

2. Uniqueness: There is at most one solution of the problem.

3. Stability: The solution depends continuously on the data.

Any problem that does not possess at least one of these requirements is said to be $i l l-$ posed. However, more attention is usually paid to the third requirement. Indeed, the existence and the uniqueness parts in the Hadamard definition are important but if they are not satisfied, they can be enforced by adding additional requirements to the solution or relaxing the notion of a solution. The requirement that the solution should depend continuously on the data is important in the sense that if one wants to approximate the solution to a problem, whose solution does not depend continuously on the data by a traditional numerical method, then one has to expect that the numerical solution becomes unstable. The computed solution thus has nothing to do with the true solution; see Engl et al. [7].

This definition is made precise with the specification of the function spaces in which the solution is sought and the boundary data are set.

As examples, we consider two Cauchy problems, problems for which the boundary data are given only on a part of the boundary of the domain. The first example is the classical example introduced by Hadamard; see [9]. The second one concerns the main subject of our thesis. 
Example 0.1. Consider the Cauchy problem for the Laplace equation:

$$
\begin{cases}\Delta u=0, & 0<x<\pi, \quad y>0, \\ u(x, 0)=0, & 0 \leq x \leq \pi, \\ \partial_{y} u(x, 0)=g_{n}(x), & 0 \leq x \leq \pi,\end{cases}
$$

where $g_{n}(x)=n^{-1} \sin n x$ for $0 \leq x \leq \pi$ and $n=1,2, \ldots$ The solution to this problem is given by

$$
u_{n}(x, y)=n^{-2} \sin n x \sinh n y .
$$

We observe that $g_{n}$ tends uniformly to zero as $n$ tends to infinity, while for fixed $y>0$, the value of $u_{n}(x, y)$ tends to infinity. Thus, the requirement that the solution depends continuously on the data does not hold.

Example 0.2. Consider the following Cauchy problem for the Helmholtz equation in the rectangle $\Omega=(0, a) \times(0, b)$ :

$$
\begin{cases}\Delta u(x, y)+k^{2} u(x, y)=0, & 0<x<a, \quad 0<y<b \\ u(x, 0)=f(x), & 0 \leq x \leq a \\ \partial_{y} u(x, 0)=g(x), & 0 \leq x \leq a \\ u(0, y)=u(a, y)=0, & 0 \leq y \leq b\end{cases}
$$

where $k$ is the wave number, $f \in L^{2}(0, a)$, and $g \in L^{2}(0, a)$ are specified Cauchy data. The solution to this problem can be obtained using separation of variables in the form

$$
u(x, y)=\sum_{n=1}^{\infty} \sin \frac{n \pi}{a} x\left(A_{n} \cosh \lambda_{n} y+\lambda_{n}{ }^{-1} B_{n} \sinh \lambda_{n} y\right),
$$

where $\lambda_{n}=\sqrt{a^{-2} n^{2} \pi^{2}-k^{2}}$ and the coefficients $A_{n}$ and $B_{n}$ are given by

$$
A_{n}=\frac{2}{a} \int_{0}^{a} f(x) \sin \frac{n \pi}{a} x d x \quad \text { and } \quad B_{n}=\frac{2}{a} \int_{0}^{a} g(x) \sin \frac{n \pi}{a} x d x .
$$

Since the estimate $\|u\|_{L^{2}(\Omega)} \leq C\left(\|f\|_{L^{2}(0, a)}+\|g\|_{L^{2}(0, a)}\right)$ cannot hold in general, the requirement that the solution depends continuously on the data does not hold and the problem is ill-posed. Note that this estimate cannot hold for any reasonable choice of norms.

More about inverse problems and ill-posed problems can be found in literature such as Groetsch [8], Isakov [11], Kaipio [12], Vogel [33], Bakushinsky and Goncharsky [13], and Lavrent'ev [24, 25], etc.

In order to obtain approximate solutions to ill-posed problems that are less sensitive to perturbations, one uses regularization methods. The regularization methods consist of reformulating the problem such that the solution to the new problem is less sensitive to the perturbations, i.e., such that the solution becomes more stable. In the literature, different regularization methods for ill-posed problems have been suggested; see for example Engl et al. [7].

There exist different versions of various regularization methods for the Cauchy problem. We mainly consider alternating iterative algorithm. 


\section{The alternating iterative method}

In this thesis we investigate the so-called alternating iterative algorithms. Introduced by V.A. Kozlov and V. Maz'ya in [21], the alternating iterative algorithms are used for solving Cauchy problem for elliptic equations. The algorithm works by iteratively changing boundary conditions until a satisfactory result is obtained. These algorithms preserve the differential equations, and every step reduces to the solution of well-posed problems for the original differential equation. The regularizing character of the algorithm is ensured solely by an appropriate choice of boundary conditions in each iteration. These methods have been applied by Kozlov et al. [22] to solve the Cauchy problem for the Laplace equation and the Lamé system. The authors also proved the convergence of the algorithms and established the regularizing properties. After that, different studies have been done using these algorithms for solving ill-posed problems originating from partial differential equations; see [1, 2, 3, 4, 27, 28, 29].

In our study, we generalize the problem in Example 0.2 as follows: Let $\Omega$ be a bounded domain in $\mathbb{R}^{n}$ with a Lipschitz boundary $\Gamma$ divided into two parts $\Gamma_{0}$ and $\Gamma_{1}$ which do not intersect and have a common Lipschitz boundary. We denote by $\nu$ the outward unit normal to the boundary $\Gamma$. We consider the following Cauchy problem for the Helmholtz equation:

$$
\begin{cases}\Delta u+k^{2} u=0 & \text { in } \Omega, \\ u=f & \text { on } \Gamma_{0}, \\ \partial_{\nu} u=g & \text { on } \Gamma_{0},\end{cases}
$$

where the wave number $k^{2}$ is a positive real constant, $\partial_{\nu}$ denotes the outward normal derivative, and $f$ and $g$ are specified Cauchy data on $\Gamma_{0}$. We want to find real solutions to the problem (1.1). In the alternating iterative algorithm described in [22], for problem (1.1), one considers the following two auxiliary problems:

$$
\begin{cases}\Delta u+k^{2} u=0 & \text { in } \quad \Omega, \\ u=f & \text { on } \Gamma_{0}, \\ \partial_{\nu} u=\eta & \text { on } \Gamma_{1},\end{cases}
$$

and

$$
\begin{cases}\Delta u+k^{2} u=0 & \text { in } \Omega, \\ \partial_{\nu} u=g & \text { on } \Gamma_{0}, \\ u=\phi & \text { on } \Gamma_{1},\end{cases}
$$

where $f$ and $g$ are the original Cauchy data as seen in (1.1). The standard alternating iterative procedure for solving the problem (1.1) is as follows:

1. The first approximation $u_{0}$ to the solution $u$ of (1.1) is obtained by solving (1.2), where $\eta$ is an arbitrary initial approximation of the normal derivative on $\Gamma_{1}$. 
2. Having constructed $u_{2 n}$, we find $u_{2 n+1}$ by solving (1.3) with $\phi=u_{2 n}$ on $\Gamma_{1}$.

3. We then find $u_{2 n+2}$ by solving (1.2) with $\eta=\partial_{\nu} u_{2 n+1}$ on $\Gamma_{1}$.

Using the problem stated in Example 0.2, we show in Paper 1; see [31], that for

$$
k^{2} \geq \pi^{2}\left(a^{-2}+(4 b)^{-2}\right)
$$

this algorithm diverges and it thus cannot be applied for large values of the constant $k^{2}$ in the Helmholtz equation. We therefore need to develop appropriate iterative methods that can be used to solve the Cauchy problem for the Helmholtz equation.

\section{Summary of papers}

\section{Paper 0}

In this paper we study the Cauchy problem for the Helmholtz equation in detail. The problem is severely ill-posed and thus challenging to solve numerically. The degree of ill-posedness for a problem is often defined in terms of the singular value decomposition of a linear operator related to the inverse problem we want to solve. Also, standard numerical methods for solving ill-posed problems are also often formulated in terms of linear operators, or matrices for discrete problems. Thus it is often useful to reformulate the Cauchy problem as a linear operator equation.

Since we are interested in studying the Cauchy problem numerically we select a concrete test case: Let the domain be $\Omega=[0,1] \times[0, L]$ and consider the following well-posed boundary value problem: Find $u(x, y)$ such that

$$
\begin{cases}\Delta u+k^{2} u=0, & 0<x<1,0<y<L, \\ u_{y}(x, 0)=0, & 0<x<1 \\ u(0, y)=u(1, y)=0, & 0<y<L \\ u(x, L)=f(x), & 0<x<1\end{cases}
$$

where $k^{2}$ is the wave number. Using this boundary value problem we define a linear operator

$$
K_{1}: f(x) \mapsto u(x, 0)=g(x) .
$$

The Cauchy problem for the Helmholtz equation is thus reformulated as a linear operator equation $K_{1} f=g$. By discretizing the above mixed boundary value problem using finite differences, we approximate the linear operator $K_{1}$ by a matrix (also denoted by $K_{1}$ ) and we are left with a linear system of equations,

$$
K_{1} F=G \text {. }
$$

The linear system $K_{1} F=G$ can be analyzed in terms of the singular value decomposition. The rate of decay of the singular values determine the degree 
of ill-posedness of the problem. In the paper we study how the singular values depend on the size of the domain, i.e. the parameter $L$, and also on the wave number $k^{2}$, and conclude that $L$ significantly influences the stability of the problem, while the wave number $k^{2}$ does not.

Further, after having reduced the Cauchy problem to a linear system of equations, $K_{1} F=G$, we demonstrate that standard regularization techniques, such as Tikhonov's method, or the conjugate gradient method, can be used for solving the problem.

\section{Paper 1}

The main idea in this paper is to introduce an artificial interior boundary $\gamma$ and a positive constant $\mu$. We then assume that

$$
\int_{\Omega}\left(|\nabla u|^{2}-k^{2} u^{2}\right) d x+\mu \int_{\gamma} u^{2} d S>0
$$

for $u \in H^{1}(\Omega)$ such that $u \neq 0$. We denote by $[u]$ and by $\left[\partial_{\nu} u\right]$ the jump of the function $u$ and the jump of the normal derivative $\partial_{\nu} u$ across $\gamma$, respectively. We propose a modified iterative algorithm that consists of solving the following boundary value problems alternatively:

$$
\begin{cases}\Delta u+k^{2} u=0 & \text { in } \quad \Omega \backslash \gamma, \\ u=f & \text { on } \Gamma_{0}, \\ \partial_{\nu} u=\eta & \text { on } \Gamma_{1}, \\ {\left[\partial_{\nu} u\right]+\mu u=\xi} & \text { on } \gamma, \\ {[u]=0} & \text { on } \gamma,\end{cases}
$$

and

$$
\begin{cases}\Delta u+k^{2} u=0 & \text { in } \quad \Omega \backslash \gamma, \\ \partial_{\nu} u=g & \text { on } \Gamma_{0}, \\ u=\phi & \text { on } \Gamma_{1}, \\ u=\varphi & \text { on } \gamma .\end{cases}
$$

The modified alternating iterative algorithm for solving (1.1) is as follows:

1. The first approximation $u_{0}$ to the solution of (1.1) is obtained by solving (2.4), where $\eta$ is an arbitrary initial approximation of the normal derivative on $\Gamma_{1}$ and $\xi$ is an arbitrary approximation of $\left[\partial_{\nu} u\right]+\mu u$ on $\gamma$.

2. Having constructed $u_{2 n}$, we find $u_{2 n+1}$ by solving (2.5) with $\phi=u_{2 n}$ on $\Gamma_{1}$ and $\varphi=u_{2 n}$ on $\gamma$.

3. We then obtain $u_{2 n+2}$ by solving the problem (2.4) with $\eta=\partial_{\nu} u_{2 n+1}$ on $\Gamma_{1}$ and $\xi=\left[\partial_{\nu} u_{2 n+1}\right]+\mu u_{2 n+1}$ on $\gamma$. 
In this paper, problems $(2.4)-(2.5)$ are solved in the weak sense. This modification thus consists of solving two well-posed mixed boundary value problems for the original equation. Since the algorithm described through auxiliary problems (2.4) and (2.5) makes sense if (2.3) holds, a sufficient condition concerning the choice of $\gamma$ and $\mu$ so that (2.3) holds is proved in this paper. It is also shown that if the positivity condition $(2.3)$ is satified, the sequence $\left(u_{n}\right)_{n=0}^{\infty}$ obtained from this procedure converges in the space $H^{1}(\Omega)$ given that the Cauchy data $f$ and $g$ belong to $H^{1 / 2}\left(\Gamma_{0}\right)$ and $H^{1 / 2}\left(\Gamma_{0}\right)^{*}$; respectively, and the initial approximations $\eta$ and $\xi$ belong to $H^{1 / 2}\left(\Gamma_{1}\right)^{*}$ and $\xi \in H^{1 / 2}(\gamma)^{*}$. In the case of inexact data, the stopping rule is suggested in Paper 2. This stopping rule is based on the stopping rule for alternating procedure proposed in [3]. This algorithm thus produce a stable sequence in the presence of noisy data.

The numerical implementation is based on solving the well-posed boundary value problems (2.4) and (2.5) using the finite difference method. This method is easy to implemente and during the computations, two matrices together with their LU decompositions are also saved which reduces the computation speed.

\section{Paper 2}

The convergence of iterative method presented in Paper 1 has been reported slow. In this paper, we demonstrate how to instead use conjugate gradient methods for accelerating the convergence. The main requirement for the formulation of these methods is the positivity condition (2.3). We thus assume first that the interior boundary $\gamma$ and the constant $\mu$ described in Paper 1 are chosen so that the positivity condition (2.3) is satified. We then present two equivalent operator formulations of problem (1.1). The first formulation corresponds to two iterations in the modified algorithm and the second to one iteration. The first one involves the operator $B$. This operator is defined through the first two iterations in the algorithm described in Paper 1 . The second one is the operator $N$ that is defined from auxiliary problem (2.4). Using auxiliary problem (2.5), we also find an adjoint operator $N^{*}$ to the operator $N$. We finally prove that the two operator equations are identical by showing that $N^{*} N=I-B$, where $I$ is the identity operator.

The convergence results for the conjugate gradient type methods in the case of exact and inexact Cauchy data proposed by Hanke in [32] are also suggested. It is also proved that the modified algorithm presented in Paper 1 can be interpreted as the Landweber iterative method under some conditions. For numerical implementation different choices of the interior boundary have been also considered. The numerical results have confirmed that the conjugate gradient methods proposed in this paper accelerate the convergence of the algorithm in Paper 1.

\section{Paper 3}

In this paper, we propose two new methods based on iterative procedure suggested in [22]. The first algorithm is a slight change of the original method. In 
Section 1, we present the original algorithm based on solving two well-posed boundary value problems with the Dirichlet and Neumann boundary conditions iteratively alternated on $\Gamma_{0}$ and $\Gamma_{1}$. We propose here to instead alternate the Dirichlet and Neumann boundary conditions on $\Gamma_{0}$ and the Robin and Dirichlet boundary conditions on $\Gamma_{1}$. In the same idea, we propose another iterative method that consists on introducing first an artificial interior boundary $\gamma$ inside $\Omega$ and a positive constant $\mu$. In this algorithm, we alternate the Dirichlet and Neumann boundary conditions on $\Gamma_{0}$ and $\Gamma_{1}$ and the Robin and Dirichlet boundary conditions on $\gamma$.

The first algorithm is described as follows: let us assume that $\mu$ is a positive constant chosen so that

$$
\int_{\Omega}\left(|\nabla u|^{2}-k^{2} u^{2}\right) d x+\mu \int_{\Gamma_{1}} u^{2} d S>0,
$$

for all $u \in H^{1}(\Omega)$, such that $u \neq 0$. Consider now the following boundary value problems

$$
\begin{cases}\Delta u+k^{2} u=0 & \text { in } \quad \Omega, \\ u=f & \text { on } \Gamma_{0}, \\ \partial_{\nu} u+\mu u=\eta & \text { on } \Gamma_{1},\end{cases}
$$

and

$$
\begin{cases}\Delta u+k^{2} u=0 & \text { in } \Omega, \\ \partial_{\nu} u=g & \text { on } \Gamma_{0}, \\ u=\phi & \text { on } \Gamma_{1} .\end{cases}
$$

Assume that $f \in H^{1 / 2}\left(\Gamma_{0}\right)$ and $g \in H^{1 / 2}\left(\Gamma_{0}\right)^{*}$ are as in (1.1). The algorithm for solving (1.1) is described as follows:

1. The first approximation $u_{0}$ is obtained by solving (2.7), where $\eta$ is an arbitrary initial approximation of the Robin condition on $\Gamma_{1}$.

2. Having constructed $u_{2 n}$, we find $u_{2 n+1}$ by solving (2.8) with $\phi=u_{2 n}$ on $\Gamma_{1}$.

3. We then obtain $u_{2 n+2}$ by solving (2.7) with $\eta=\partial_{\nu} u_{2 n+1}+\mu u_{2 n+1}$ on $\Gamma_{1}$.

The convergence of this algorithm follows from the convergence of the algorithm in [22]. The main idea in the second algorithm is to introduce an artificial interior boundary $\gamma$ and a positive constant $\mu$ such that

$$
\int_{\Omega}\left(|\nabla u|^{2}-k^{2} u^{2}\right) d x+\mu \int_{\gamma} u^{2} d S>0
$$

for all $u \in H^{1}(\Omega), u \neq 0$. The new alternating procedure consists of solving the 
following two well-posed boundary value problems

$$
\begin{cases}\Delta u+k^{2} u=0 & \text { in } \Omega \backslash \gamma, \\ u=f & \text { on } \Gamma_{0}, \\ \partial_{\nu} u=\eta & \text { on } \Gamma_{1}, \\ \partial_{\nu} u+\mu u=\xi & \text { on } \gamma,\end{cases}
$$

and

$$
\begin{cases}\Delta u+k^{2} u=0 & \text { in } \Omega \backslash \gamma, \\ \partial_{\nu} u=g & \text { on } \Gamma_{0}, \\ u=\phi & \text { on } \Gamma_{1}, \\ u=\psi & \text { on } \gamma .\end{cases}
$$

Assume that $f \in H^{1 / 2}\left(\Gamma_{0}\right)$ and $g \in H^{1 / 2}\left(\Gamma_{0}\right)^{*}$ are as in (1.1). The algorithm for solving (1.1) is described as follows:

1. The first approximation $u_{0}$ is obtained by solving (2.10), where $\eta$ is an arbitrary approximation of the Neumann boundary condition on $\Gamma_{1}$ and $\xi$ is an arbitrary initial approximation of the Robin condition on $\gamma$.

2. Having constructed $u_{2 n}$, we find $u_{2 n+1}$ by solving (2.11) with $\phi=u_{2 n}$ on $\Gamma_{1}$ and $\psi=u_{2 n}$ on $\gamma$.

3. We then obtain $u_{2 n+2}$ by solving the problem (2.10) with $\eta=\partial_{\nu} u_{2 n+1}$ on $\Gamma_{1}$ and $\xi=\partial_{\nu} u_{2 n+1}+\mu u_{2 n+1}$ on $\Gamma_{1}$.

While we introduce jump conditions in Paper 1, we instead here solve the wellposed problems in the domain between two boundaries $\gamma$ and $\Gamma$. This reduces the computation since the number of unknowns reduces during the discretization of the problems. A sufficient condition for the choices of the interior boundary $\gamma$ and $\mu$ is discussed for a one and a two-dimensional cases. It is shown that the constant $\mu$ is chosen sufficiently large so that (2.9) holds. 


\section{References}

[1] S. Avdonin, D. Maxwell, M. Truffer and M. Stuefer, An iterative scheme for determining glacier velocities and stresses, Journal of Glaciology, $\mathbf{5 4}$ (2008), no. 188, 888-898.

[2] S. Avdonin, V. Kozlov, D. Maxwell and M. Truffer, Iterative methods for solving a nonlinear boundary inverse problem in glaciology, J. Inv. Ill-Posed Problems, 17 (2009), 239-258.

[3] G. Bastay, T. Johansson, V.A. Kozlov and D. Lesnic, An alternating method for the stationary Stokes system, ZAMM, Z. Angew. Math. Mech., 86 (2006), no. 4, 268-280.

[4] R. Chapko and B.T. Johansson, An alternating potential-based approach to the Cauchy problem for the Laplace equation in a planar domain with a cut, Computational Methods in Applied Mathematics, 8 (2008), no. 4, $315-335$.

[5] D. Colton and R. Kress, Inverse Acoustic and Electromagnetic Scattering Theory, Second edition, Springer-Verlag, 1998.

[6] A.M. Denisov, Elements of the Theory of Inverse Problems, VSP BV, 1999.

[7] H.W. Engl, M. Hanke and A. Neubauer, Regularization of Inverse Problems, Kluwer Academic Publishers, 2000.

[8] C.W. Groetsch, Inverse Problems in Mathematical Sciences, Vieweg, 1993.

[9] J. Hadamard, Lectures on Cauchy's Problem in Linear Partial Differential Equations, Dover Publications, New York, 1952.

[10] P.C. Hansen, Discrete Inverse Problems: Insight and Algorithms, SIAM, 2010.

[11] V. Isakov, Inverse Problems for Partial Differential Equations, SpringerVerlag, 1998.

[12] J. Kaipio and E. Somersalo, Statistical and Computational Inverse Problems, Springer-Verlag, 2005.

[13] A. Bakushinsky and A. Goncharsky, Ill-Posed Problems: Theory and Applications, Kluwer Academic Publishers, 1994.

[14] J.T. Chen and F.C. Wong, Dual formulation of multiple reciprocity method for the acoustic mode of a cavity with a thin partition, Journal of Sound and Vibration, 217(1):75-95, 1998.

[15] T. Regińska and K. Regiński, Approximate solution of a Cauchy problem for the Helmholtz equation, Inverse Problems, 22(3):975-989, 2006. 
[16] J.D. Shea, P. Kosmas, S.C. Hagness, and B.D. Van Veen, Threedimensional microwave imaging of realistic numerical breast phantoms via a multiple frequency inverse scattering technique, Medical Physics, 37(8):4210-4226, 2010.

[17] T. Delillo, V. Isakov, N. Valdivia, and L. Wang, The detection of the source of acoustical noise in two dimensions, SIAM J. Appl. Math., 61(6):21042121, 2001.

[18] G.J. Fix and S.P. Marin, Variational methods for underwater acoustic problems, J. Comput. Phys., 28:253-270, 1978.

[19] A. Kirsch, An Introduction to the Mathematical Theory of Inverse Problems, Springer-Verlag, 1996.

[20] T. Delillo, V. Isakov, N. Valdivia, and L. Wang, The detection of surface vibrations from interior acoustical pressure, Inverse Problems 19 (2003), $507-524$

[21] V.A. Kozlov and V.G. Maz'ya, On iterative procedures for solving illposed boundary value problems that preserve differential equations, Algebra i Analiz, 192 (1989), 1207-1228.

[22] V.A. Kozlov, V.G. Maz'ya and A.V. Fomin, An iterative method for solving the Cauchy problem for elliptic equations, Comput. Maths. Math. Phys., 31 (1991), no. 1, 46-52.

[23] C. Langrenne, M. Melon and A. Garcia, Boundary element method for the acoustic characterization of a machine in bounded noisy environment, J. Acoust. Soc. Am., 121 (2007), no. 5, 2750-2757.

[24] M.M. Lavrent'ev, V.G. Romanov and S.P. Shishatskii, Ill-Posed Problems of Mathematical Physics and Analysis, American Mathematical Society, 1986 .

[25] M.M. Lavrent'ev, Some Improperly Posed Problems of Mathematical Physics, Springer-Verlag, 1967.

[26] M.M. Lavrent'ev and L.Ya. Savel'ev, Linear Operators and Ill-Posed Problems, Translated form Russian by Nauka Publishers Moscow, 1995.

[27] D. Lesnic, L. Elliot and D.B. Ingham, An alternating boundary element method for solving numerically the Cauchy problems for the Laplace equation, Engineering Analysis with Boundary Elements, 20 (1997), no. 2, 123133.

[28] D. Lesnic, L. Elliot and D.B. Ingham, An alternating boundary element method for solving Cauchy problems for the biharmonic equation, Inverse Problems in Engineering, 5 (1997), no. 2, 145-168. 
[29] L. Marin, L. Elliott, P.J. Heggs, D.B. Ingham, D. Lesnic and X. Wen, An alternating iterative algorithm for the Cauchy problem associated to the Helmholtz equation, Comput. Math. Appl. Mech. Eng., 192 (2003), 709722.

[30] M. Hanke, The minimal error conjugate gradient method is a regularization method, Proceedings of the AMS, 123 (1995), no. 11.

[31] F. Berntsson, V.A. Kozlov, L. Mpinganzima, and B.O. Turesson, An alternating iterative procedure for the Cauchy problem for the Helmholtz equation, Inverse Problems in Science and Engineering, 22 (2014), no. 1, 45-62.

[32] M. Hanke, Conjugate Gradient Type Methods for Ill-Posed Problems, Longman Scientific and Technical, Harlow, Essex, 1995.

[33] C.R. Vogel, Computational Methods for Inverse Problems, SIAM, 2002. 


\section{Papers}

The articles associated with this thesis have been removed for copyright reasons. For more details about these see:

http://urn.kb.se/resolve?urn=urn:nbn:se:liu:diva-105879 